\title{
Characterization of Impulse Radio Intrabody Communication System for Wireless Body Area Networks
}

\author{
Zibo Cai $^{1} \cdot$ MirHojjat Seyedi $^{1} \cdot$ Weiwei Zhang $^{2} \cdot$ Francois Rivet $^{3} \cdot$ Daniel T. H. Lai $^{1}$
}

Received: 17 August 2015/ Accepted: 26 May 2016/Published online: 6 January 2017

(C) Taiwanese Society of Biomedical Engineering 2017

\begin{abstract}
Intrabody communication (IBC) is a promising data communication technique for body area networks. This short-distance communication approach uses human body tissue as the medium of signal propagation. IBC is defined as one of the physical layers for the new IEEE 802.15.6 or wireless body area network (WBAN) standard, which can provide a suitable data rate for real-time physiological data communication while consuming lower power compared to that of radio-frequency protocols such as Bluetooth. In this paper, impulse radio (IR) IBC (IRIBC) is examined using a field-programmable gate array (FPGA) implementation of an IBC system. A carrier-free pulse position modulation (PPM) scheme is implemented using an IBC transmitter in an FPGA board. PPM is a modulation technique that uses time-based pulse characteristics to encode data based on IR concepts. The transmission performance of the scheme was evaluated through
\end{abstract}

Zibo Cai

zibocai@gmail.com

MirHojjat Seyedi

mirhojjat.seyedi@live.vu.edu.au

Weiwei Zhang

zdw@ustc.edu

Francois Rivet

francois.rivet@ims-bordeaux.fr

Daniel T. H. Lai

daniel.lai@vu.edu.au

1 College of Engineering and Science, Victoria University, Melbourne, Australia

2 Jiangxi Engineering Laboratory for Optoelectronics Testing Technology, Nanchang Hang Kong University, Nanchang, China

3 IMS Laboratory, University of Bordeaux, Bordeaux, France signal propagation measurements of the human arm using 4- and 8-PPM transmitters, respectively. 4 or 8 is the number of symbols during modulations. It was found that the received signal-to-noise ratio (SNR) decreases approximately $8.0 \mathrm{~dB}$ for a range of arm distances $(5-50 \mathrm{~cm})$ between the transmitter and receiver electrodes with constant noise power and various signal amplitudes. The SNR for the 4-PPM scheme is approximately $2 \mathrm{~dB}$ higher than that for the 8-PPM one. In addition, the bit error rate (BER) is theoretically analyzed for the human body channel with additive white Gaussian noise. The 4and 8-PPM IBC systems have average BER values of $10^{-5}$ and $10^{-10}$, respectively. The results indicate the superiority of the 8-PPM scheme compared to the 4-PPM one when implementing the IBC system. The performance evaluation of the proposed IBC system will improve further IBC transceiver design.

Keywords Field-programmable gate array (FPGA) . Impulse radio (IR) - Intrabody communication (IBC) . Pulse position modulation (PPM) - Transmitter design . Wireless body area network (WBAN)

\section{Introduction}

The development focus of communication networks is shifting from wireless local area networks (WLAN) to wireless personal area networks (WPAN). Wireless body area networks (WBAN) are a subset of WPAN that is primarily used for biomedical applications [1]. For WBAN, sensors in a wireless network are worn by or implanted into a human user. Wearable sensor networks are employed for on-body communication to continually monitor a person's health during daily activities [2], whereas implant sensor 
networks transmit physical and physiological data from inside the human body.

WBAN operates in a frequency range of $1.0 \mathrm{MHz}$ to 10.0 GHz for long-term healthcare monitoring [3]. WBAN communication requirements are specified by the Physical (PHY) and medium access control (MAC) layer definitions of IEEE 802.15.6, which was released in February, 2012 [4]. The IEEE 802.15.6 standard ratified three PHY layers: narrow band (NB) layer, ultra-wide band (UWB) layer, and human body communication (HBC) layer. According to the standard, HBC has a $21.0-\mathrm{MHz}$ center frequency and usually works below $50 \mathrm{MHz}$ [5]. It is a promising candidate for wearable on-body communication, because the high conductivity of the human body in this frequency band leads to lower propagation loss [6]. HBC has also been referred to as intrabody communication (IBC) [7] and body channel communication (BBC) [8]. The present study uses the term "IBC".

IBC normally employs frequency shift keying (FSK) or on-off keying (OOK) modulation schemes, and thus achieves a relatively low data rate (dozens of kbps) [8]. Due to the requirement of two different frequencies for binary digital data representation, FSK occupies double the spectrum width compared to that of OOK. Pulse position modulation (PPM) uses pulse to represent the data symbols based on impulse radio (IR) concepts [9]. IR involves transmitting a very short pulse with no radio frequency modulation, making it suitable for low-power-consumption, high-data-rate, and short-distance communication systems [1]. Since PPM is a time-based technique, it has a lower possibility of false detection compared to that of OOK, which is a shape-based modulation scheme. Because PPM uses pulses with similar amplitudes, the detection of channel noise is simpler than that for OOK. As only one time slot is active during data transmission, PPM decreases the average power requirement uses a larger bandwidth. Compared with OOK modulation, PPM has lower average power, higher peak power, and higher signal-to-noise ratio (SNR) performance in HBC systems [10]. The wide bandwidth of the PPM scheme is adequate for IBC with a center frequency of $21.0 \mathrm{MHz}$.

The OOK modulation scheme was utilized in a near-field IBC prototype to achieve a simple structure and cost-efficient design [7]. Following several attempts to design energy-efficient IBC transceivers, a baseband system for intrabody channel communication was proposed by Zhang et al. [11]. An FSK modulation scheme was used in their proposed galvanic coupling IBC system. An OOK transceiver was proposed by Tengfei et al. [12] for WBAN that uses a field-programmable gate array (FPGA) implementation using a 30-MHz pulse carrier. The achieved data rate of their OOK-IBC system was 2 Mbps. Recently, an IBC transceiver based on the IR technique was developed by
Shikada et al. [13]. They used an OOK modulation scheme for designing their capacitive coupling IBC transmitter and receiver. The performance of a PPM scheme was evaluated by Anzai et al. [10] for a UWB-IR transmission system operated in the frequency band $3.4-4.8 \mathrm{GHz}$. The proposed system was for radio frequency (RF) communication rather than IBC, which operates at a lower frequency (less than $50 \mathrm{MHz}$ ) [12]. To the best of our knowledge, the PPM scheme has not been used for IBC.

In this study, we demonstrate an IR-IBC transmitter structure using a carrier-free PPM scheme on an FPGA platform for galvanic coupling applications. The proposed system is experimentally evaluated and a channel modeling analysis is suggested for the IBC. Results of signal propagation and communication performance based on human arm measurements with varying distance between the onbody transmitter and the receiver are presented. We examine the characteristics of the proposed IBC system, such as path loss, noise, SNR, and bit error rate (BER) through the human arm.

\section{IBC System Design}

\subsection{IBC Coupling Method}

The principle of IBC is based on the transmission of coupled electric fields to the body using human tissue [14]. Signal coupling to the body can be classified into two conceptually different approaches: capacitive coupling (electric field) and galvanic coupling [15]. In the former, the forward signal path is established through the body while the return path is connected through earth ground. In the latter, the signal is differentially coupled into the human body by a pair of coupler electrodes and sensed by a pair of receiver electrodes. Capacitive coupling is affected by environmental interference and noise (due to the use of earth ground as the return path), which can influence the accuracy of the transmitted data for practical WBAN deployments. It is therefore of interest to improve data rates with galvanic coupling methods, since they are less susceptible to environmental effects (current predominantly flows through tissues). Therefore, the galvanic approach was used to test the communication system in our study.

\subsection{IBC PPM System}

The significance of developing an IBC system lies in its vital role in transmitting human physiological parameters in healthcare monitoring. The IBC transmitter should have acceptable simplicity and a high data rate, and be energy efficient. An optimal modulation scheme should address the above factors. 
PPM is one of most popular orthogonal signal modulation techniques for both analog and digital signal transmission. It has been used in IR-type signal propagation, which is suitable for WBAN communication systems because of its low power consumption, carrier-less transmission, energy efficiency, and anti-jamming capability. Since PPM uses pulse positions to encode the original data signal, it has efficient transmit power and low BER. The changes in pulse polarity with time due to PPM break the regular intervals in the transmission, and therefore smoothen the overall spectrum by diminishing the line spectrum components [16]. The wide bandwidth of the PPM scheme can be implemented without an external carrier signal [17]. In this work, the data is modulated by PPM and transmitted through the arm as a pulse train.

Figure 1 describes the general structure of the PPM transmitter in the IBC method, where the output of the PPM transmitter is directly connected to the human body [18]. In the figure, the output data from on-body sensors are saved to the Data Sequence block. The Data Sequence's output is determined by the output of Counter_2 and flows to the comparator. The comparator consists of a logic AND gate, which directs the data toward the human body. Analytically, the PPM signal, $s(t)$, can be mathematically defined as:
$s(t)=\sum_{k=0}^{K} p\left(t-b_{k} \frac{T}{2}-k T\right)$

where $p$ is the pulse waveform and $T$ represents the symbol period. It is assumed that the values of $b^{k}$ for the $k^{\text {th }}$ transmitted bit are ' 0 ' or ' 1 '.

In PPM techniques, binary N-bit data are mapped into one of $\mathrm{L}$ possible symbol positions, where $\mathrm{L}=2^{\mathrm{N}}$. L-bit PPM-modulated-signal-transmitted bit information is $\log _{2-}$ L. It is more common to share a communication channel using time division multiplexing, where users (sensors) transmit at fixed time intervals, i.e., time slots. In a PPM time slot, one slice is occupied by a pulse represented by each symbol and $(\mathrm{L}-1)$ slices are empty. The data are encoded by the position of the pulse in a time slot. Table 1 shows 4 and 8 PPM symbol modulations based on the mapping of 2- and 3-bit words, respectively.

\subsection{System Design}

The block diagram of proposed PPM IBC transmitter implemented on an FPGA platform is shown in Fig. 1. This IBC transmitter circuit architecture generates a square wave digital signal for propagation through the human body. The VHSIC (very high speed integrated circuit) hardware
Fig. 1 Simplified architecture of IBC PPM transmitter

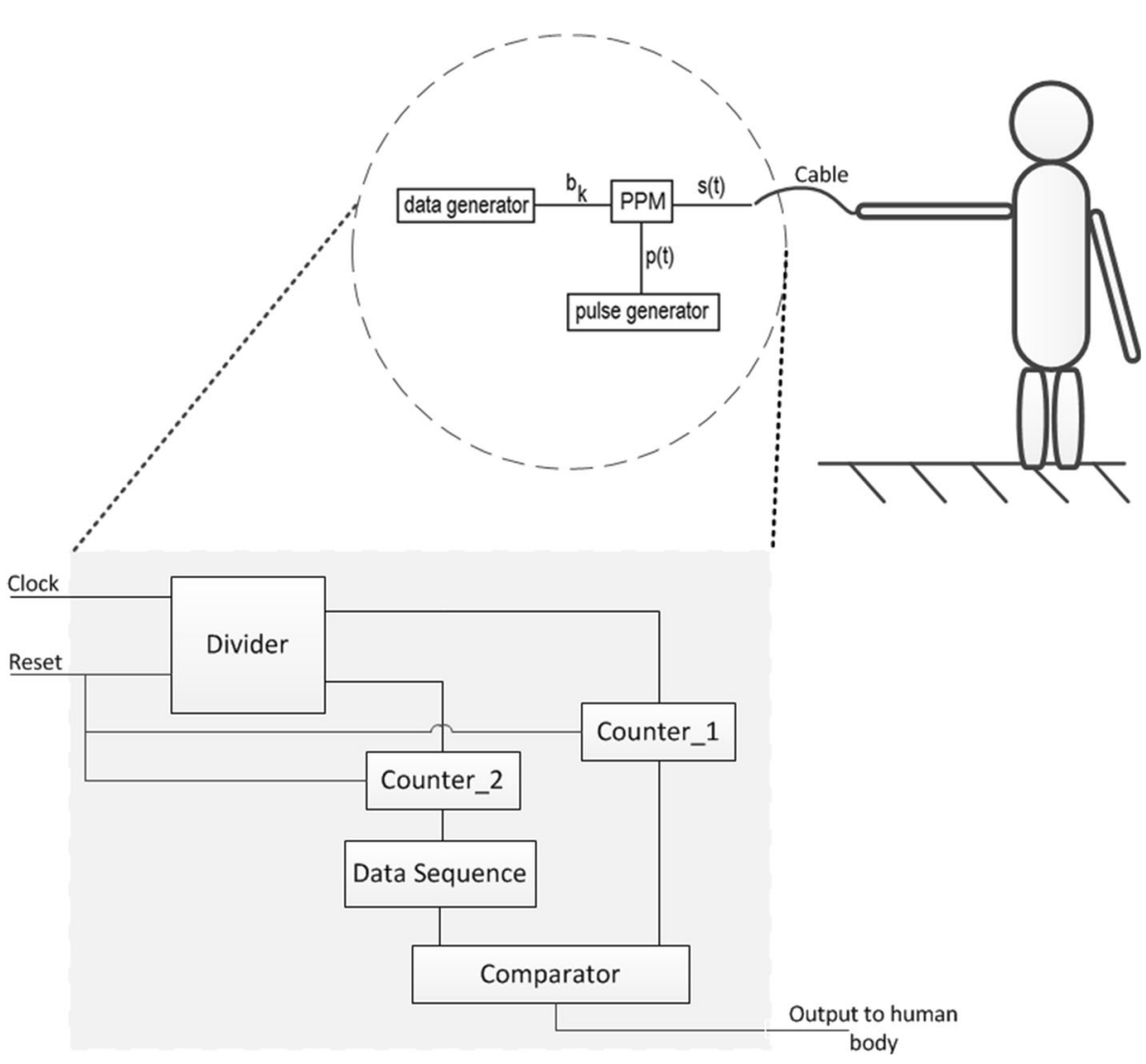


Table 1 Mapping of 2- and 3-Bit words into 4 and 8 PPM symbols

\begin{tabular}{llll}
\hline 4 PPM & & 8 PPM & \\
\cline { 4 - 4 } $\mathrm{N}=2$ & $\mathrm{~L}=4$ & $\mathrm{~N}=3$ & $\mathrm{~L}=8$ \\
\hline 00 & 1000 & 000 & 10000000 \\
01 & 0100 & 001 & 01000000 \\
10 & 0010 & 010 & 00100000 \\
11 & 0001 & 011 & 00010000 \\
N/A & & 100 & 00001000 \\
& & 101 & 00000100 \\
& & 110 & 00000010 \\
& & 111 & 00000001 \\
\hline
\end{tabular}

description language was utilized for the circuit program development.

For low power consumption, the system design requires a simple structure. The five main components in our transmitter architecture are: a comparator, a divider, two counters, and a data sequence block. The input pulse is defined as the clock frequency of the FPGA board due to the carrier-free PPM scheme implementation (no external signal used as a carrier). This clock frequency is divided by the divider block into two different frequencies based on the multi-pulse position modulation requirement. These two types of frequency pulse go through the counters, where Counter_1 controls time slot distribution and Counter_2 divides each time slot into L slices. The block following Counter_2 is data sequence block. It encodes the pulse position by slice. The comparator compares the output of Counter_1 and the data sequence. The output signal of the transmitter to the human body is ' 1 ' if they are equal value; otherwise, it is ' 0 '.

Figure 2 shows the symbol period structure of the proposed transmitter $(L=4$ and 8$)$. In a time frame for the 8-PPM transmitter, it is assumed that 64 slices are transmitted. Each time frame is divided into 8 time slots, each of which contains 8 time slices encoding 3 bits of data. It is worth noting that for this work, only 1 of 8 slices is transmitted in each time slot (i.e., 8 slices per frame). The clock frequency of the FPGA board is divided by 16 and 64 to run the counters. The position of the active slice is selected by the programmable data sequence block (data generator), and is transmitted as digital data through the body communication channel.

\section{Empirical Measurements}

\subsection{Measurement Setup}

The proposed circuit of the IBC system (see Fig. 3) was built on a Xilinx FPGA board (Virtex5-XC5VLX50T Genesys, Digilent Inc., WA) with a clock frequency of

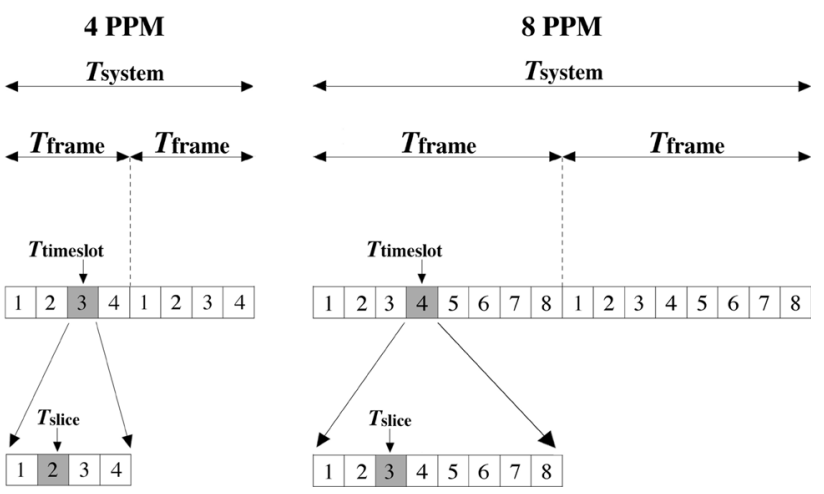

Fig. 2 4- and 8-PPM transmitter output patterns

$100 \mathrm{MHz}$. The FPGA board was powered by a battery to isolate the transmitter from mains power $(50 \mathrm{~Hz})$ coupling effects. In the measurement setup, an oscilloscope (MSO 5204 Mixed Signal Oscilloscope, Tektronix Inc., OR) and a spectrum analyzer (NS-30, LIG-NEX1 Inc., Korea) were utilized to detect the communicated signal through the body. A pair of commercial self-adhesive $\mathrm{Ag} / \mathrm{AgCl}$ single electrodes (Noraxon Inc., AZ) was used as wearable transmitter and receiver electrodes. Galvanic effects and the skin response during electrode use have negligible effects on the results $[8,32]$. For safety, the transmitter signal amplitude was kept at less than $1 \mathrm{~V}$. Although higher transmission amplitude would increase SNR and hence capacity, it was not applied for safety reasons [19]. The measured output amplitude of the battery-powered FPGA was $650 \mathrm{mV}$.

\subsection{Protocol}

Prior to performing empirical measurements on the human body, we obtained ethics approval from our university's Ethics Committee (VUHREC). We chose two healthy male

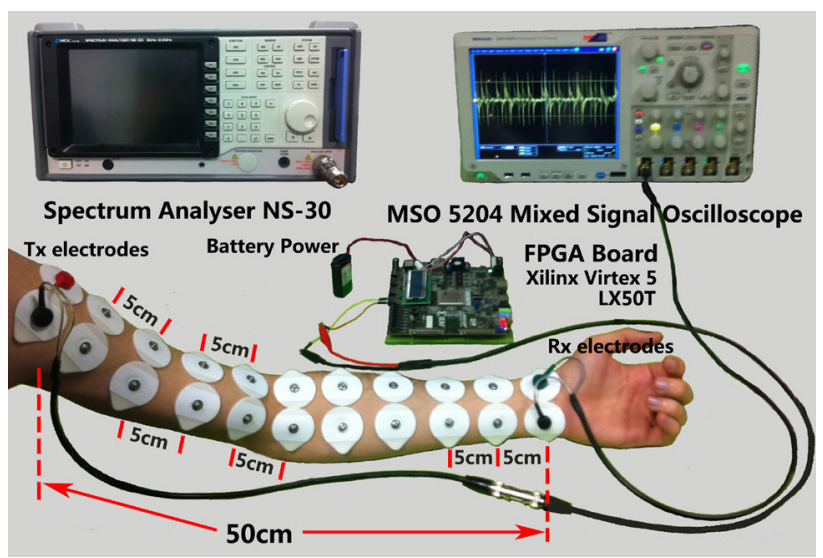

Fig. 3 Measurement setup and protocol of galvanic coupling IBC using FPGA board, transmitter and receiver electrodes, spectrum analyzer, and oscilloscope 
volunteers to participate in our experiments. The subjects were 31 and 30 years old, 179 and $182 \mathrm{~cm}$ tall, and 67 and $75 \mathrm{~kg}$ in weight, respectively.

Figure 3 shows the measurement setup of the galvanic coupling IBC using the FPGA board. In all experiments, the transmitter and receiver electrodes were attached on the subject's left arm. The transmitter and receiver electrodes were initially $5 \mathrm{~cm}$ apart. The receiver electrodes were moved away from the transmitter electrodes in $5-\mathrm{cm}$ increments (from 5 to $50 \mathrm{~cm}$ ) while the location of the transmitter electrodes was fixed. New receiver electrodes were used for each measurement. It was assumed that the human arm was composed of uniform body tissue. Additionally, the muscular effects were ignored in our measurements [20]. Each 5-cm on-body distance between the transmitter and receiver electrodes was modeled as a natural attenuator. We kept the receiver electrodes close to the wrist and changed the location of the transmitter electrodes on the left arm (see Fig. 3). The inter-electrode distance was fixed at $3.8 \mathrm{~cm}$ since the electrode radius was $1.9 \mathrm{~cm}$. The subjects were asked to stand and hold their left arm horizontally (parallel to the ground). The distances between the arm and the ground were 129 and $138 \mathrm{~cm}$ for the two subjects, respectively. The measurements for each position were repeated three times over three different days (at 10 am). The average data are reported.

\section{Results and Discussion}

\subsection{IBC Signals (Time and Frequency Domains)}

The baseband signal was modulated using PPM to transmit digital data through the arm channel using IBC. The characteristics of the IBC transmitter were measured using the oscilloscope. Figure 4a shows the output waveform of the 4-PPM transmitter. The duration of each slot is $640 \mathrm{~ns}$ (four slices of 160-ns duration). Therefore, the maximum data rate of this transmitter is $1.56 \mathrm{Mb} / \mathrm{s}$ when one bit is encoded in each time slot. The voltage amplitude of the output signal utilizing the 4-PPM transmitter is approximately $650 \mathrm{mV}$. Figure $4 \mathrm{~b}$ shows the received signal from the human body, whose amplitude is $13 \mathrm{mV}$. The calculated signal attenuation is around $34 \mathrm{~dB}$. For the second test subject, the attenuation was $31.5 \mathrm{~dB}$.

The characteristics of the IBC transmitter were measured using a spectrum analyzer. Figure $4 \mathrm{c}$ shows the transmission power spectra of the 4- and 8-PPM schemes. The main power of the 4-PPM scheme has a peak at $2.5 \mathrm{MHz}$ whereas that of the 8-PPM one is around 1.5 MHz. The transmitted power value of the 4-PPM scheme is always higher than that of the 8-PPM one even at the minimum values of both curves. The 4-PPM scheme has higher average power (around $15 \mathrm{~dB}$ higher) than that of the 8-PPM scheme in the $0-25 \mathrm{MHz}$ range.

The amplitude of the signal decreased due to path loss during human body (dielectric material) propagation. Figure 5 shows a comparison between the original signal and the received signal after $50-\mathrm{cm}$ arm tissue transmission. Attenuations of 41 and $37 \mathrm{~dB}$ were obtained using the 4and 8-PPM schemes, respectively. The power of the 4-PPM signal decreased from 6 to $47 \mathrm{dBm}$. The path loss of the 8-PPM signal power was between 16 and $53 \mathrm{dBm}$. Both curves started to become distorted when the transmission frequency was less than 17.5 MHz. Since the signal power is weaker at above $17.5 \mathrm{MHz}$, the propagation bandwidth of both modulation schemes should be between 0.5 and $17.5 \mathrm{MHz}$, which is within the IBC standard range [4]. It is worth noting that the frequency spectrum of the data after transmission through the body shows similar behavior to that reported by Takumi et al. [21]. This demonstrates that the PPM scheme has lower attenuation and higher SNR comparing to RF communication in the operation band of the IBC system.

\subsection{Propagation Characteristics}

The signal at the receiver can be expressed based on the friis equation $[22,23]$ :

$P_{R X}=P_{T X}+G_{T X}+G_{R X}-P_{P L}$

where $P_{R x}, P_{T x}, G_{T x}, G_{R x}$, and $P_{P L}$ are the received signal, transmitted signal, gain of electrodes on the transmitter side, gain of electrodes on the receiver side, and path loss, respectively.

If the path loss between the transmitter and receiver electrodes is modeled taking into account distance according to a semi-empirical power decay law [24, 25], the on-body path loss can be calculated as:

$P_{P L}(d)=P_{P L 0}+10 n \log _{10}\left(\frac{d}{d_{0}}\right)$

where $d$ is the distance between the transmitter and receiver electrodes, $P_{P L O}$ is the reference path loss at distance $d_{0}=5 \mathrm{~cm}$, and $n$ is the path loss exponent ( $=2$ in free space). The value of fitting coefficient $n$ is based on the communication channel.

The on-body path loss characteristic was formulated based on the path loss as a function of distance. Figure $6 \mathrm{a}$ shows the model fitting of the body channel loss over the on-body channel distance. The results demonstrate that an increase in channel length led to an increase in path loss. Our curve fitting measures that the path loss exponent $n$ equals 0.75 . The value of path loss exponent $n$ in our work is compared with those reported in previous studies in Table 2. 
Fig. 4 a Waveform of 4-PPM transmitter output. b Detected time-domain 4-PPM signal from on-body electrodes at $50-\mathrm{cm}$ distance. c Frequency-domain output signals of 4- and 8-PPM IBC transmitter $\left(T_{\mathrm{x}}\right)$ in

0-25 $\mathrm{MHz}$ range
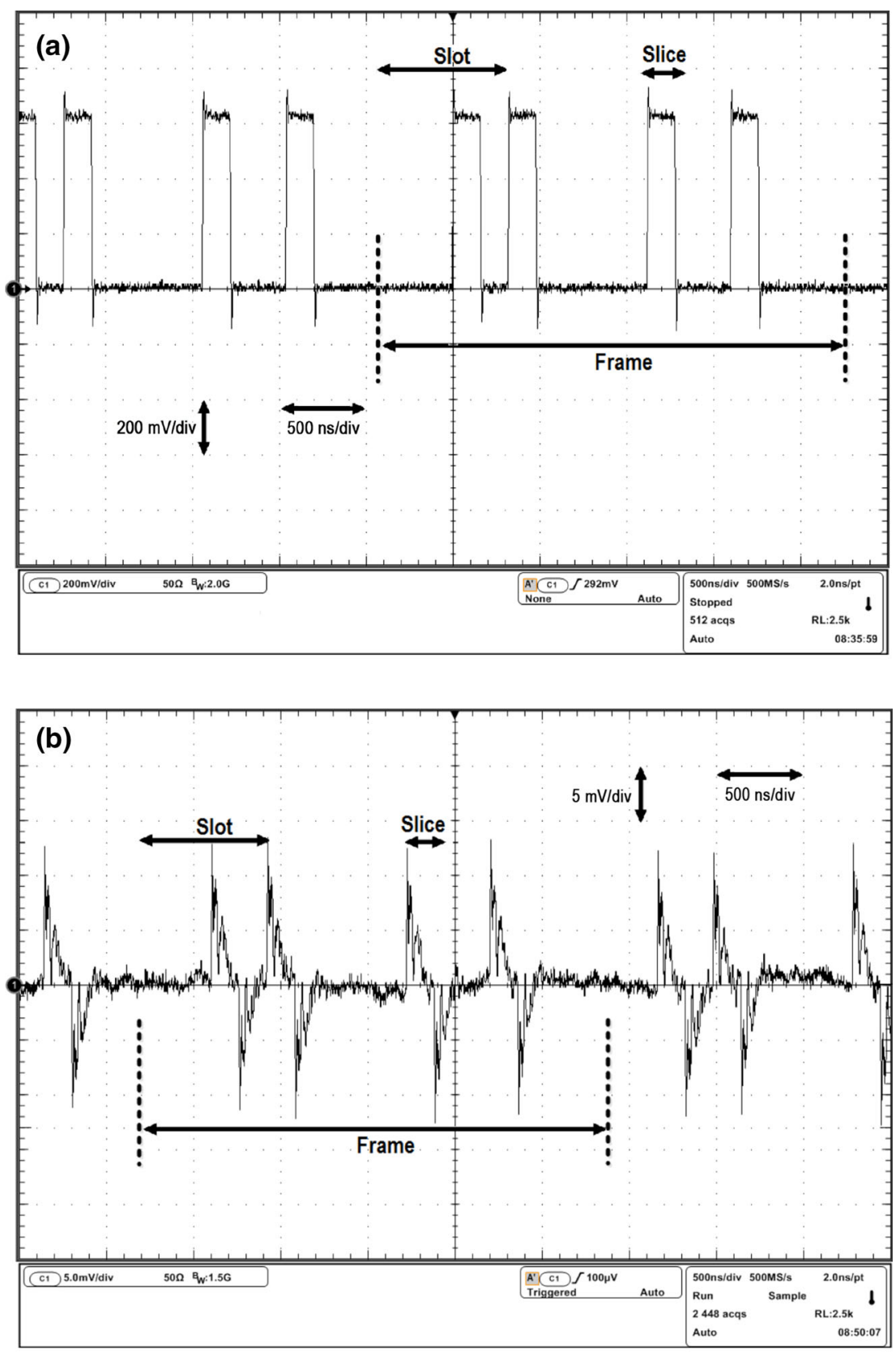

(c)

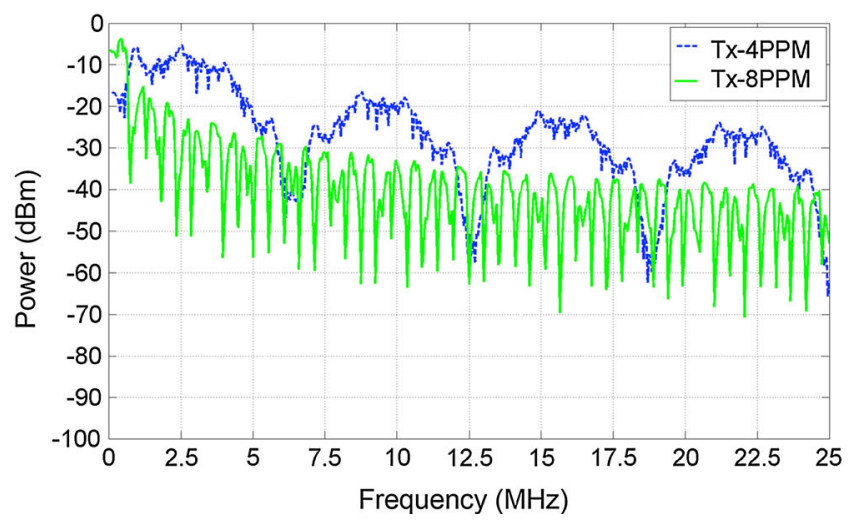



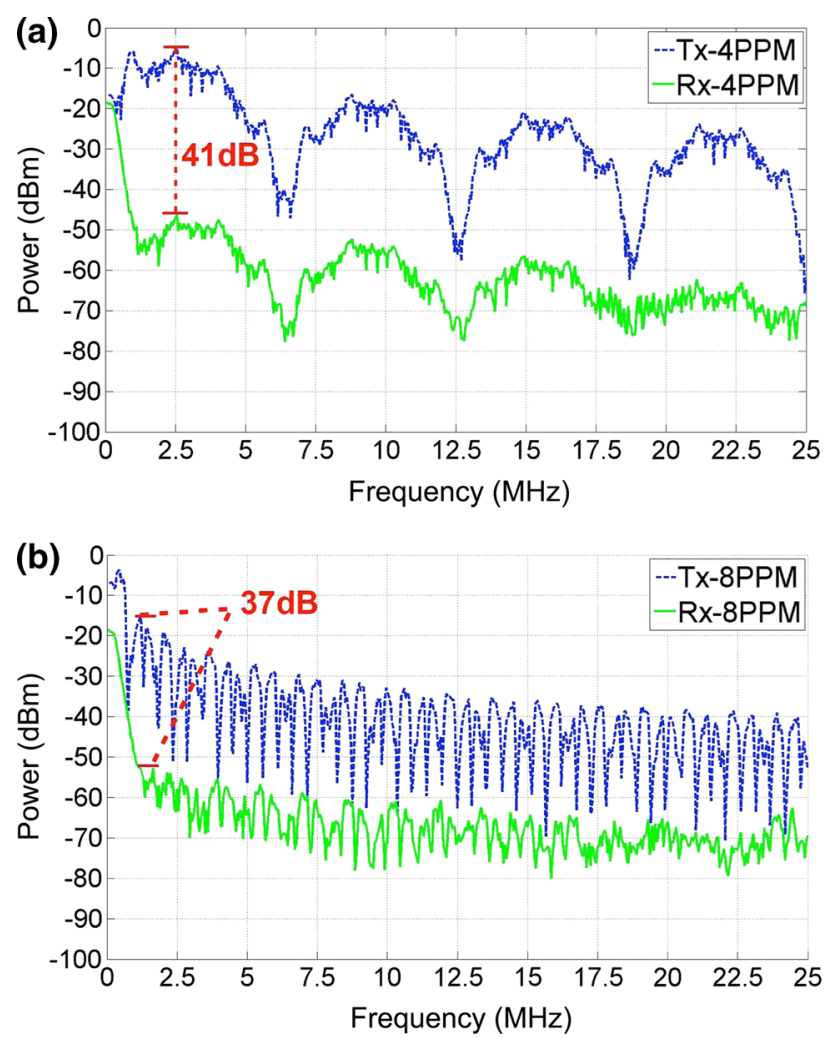

Fig. 5 Comparison of IBC signal spectra. Output of IBC transmitter (Tx) before crossing through body (blue) and output of IBC receiver (Rx) after propagating through body (green) for a 4-PPM and b 8PPM schemes below $25 \mathrm{MHz}$. Transmission distance is $50 \mathrm{~cm}$

The linear regression line was fitted using the least squares method to obtain the value of the fitting coefficient and the operational frequency. Figure $6 \mathrm{~b}$ shows the linear regression of path loss exponent $n$. The fit is good since the linear correlation coefficient, $R$, is greater than 0.9. The quantity $R$ measures the strength and direction of a linear relationship between variables.

\subsection{Signal Propagation Noise}

The received signal was assumed to be corrupted by additive white Gaussian noise (AWGN) [30]. To measure the amplitude of the received signal and noise in our IBC system, an oscilloscope was used. Figure 7 shows the received signal after human arm transmission. As shown in the figure, the signal amplitude decreases with increasing channel length but the noise remains constant.

Figure 8 depicts the received signal and measured noise amplitudes for 4- and 8-PPM schemes, respectively, for the left arm. The distance between the transmitter and receiver electrodes was varied from 5 to $50 \mathrm{~cm}$. The noise amplitude curve shows a constant average of $4 \mathrm{mV}$ across the various distances. Additionally, the curves of signal amplitude are linearly decreasing from 5 to $50 \mathrm{~cm}$; the

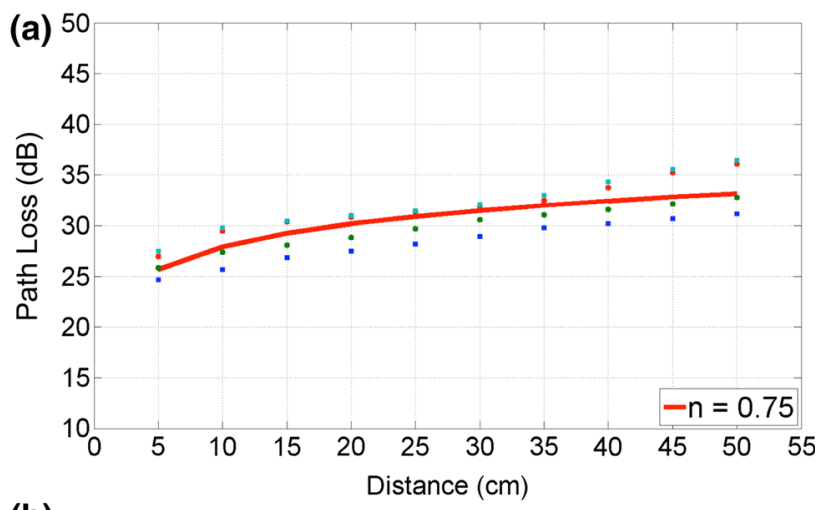

(b)

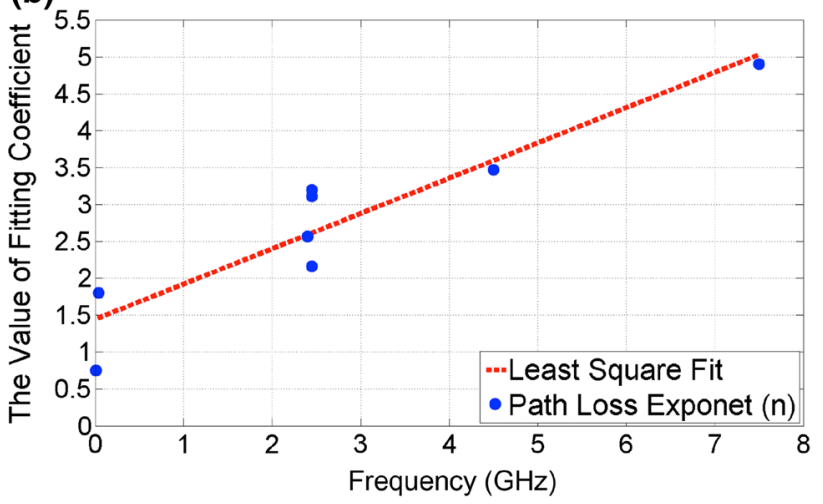

Fig. 6 a Path loss versus distance and b real path loss exponent obtained using least squares fitting

voltage of the 4-PPM scheme drops from 35 to $17.5 \mathrm{mV}$, and that of the 8-PPM scheme drops from 30 to $15 \mathrm{mV}$. The noise characteristics follow the Gaussian distribution [31]. The noise value in our experiment was found to be $4 \mathrm{mV}$ or $15 \mathrm{dBm}$. To evaluate the performance of the developed system, an analytical channel model of the body channel was used for signal characteristics investigation, as shown in Fig. 9. The human body channel distance between the transmitter and the receiver as well as external noise sources are modeled. Following Wegmueller [32], we used an AWGN channel model as the IBC transmission channel. As the experimental environment was similar for IBC measurements with different channel lengths, the power spectral density of AWGN is assumed to be constant during the measurements.

\subsection{Communication Performance and BER Evaluation}

For short-distance wireless transmission systems, communication performance degrades with increasing noise power [10]. Based on a simulation of the theoretical model, the SNR was calculated considering constant noise power $(-15 \mathrm{dBm})$. Figure 10a shows the SNR variation of the received signal for various distances $(5-50 \mathrm{~cm})$ through the left arm of subjects 1 and 2 using the 4- and 8-PPM 
Table 2 Comparison of pathloss exponent at various transmission frequencies
Fig. 7 Received signal after propagating through body channel over distances of 10 , 15,25 , and $45 \mathrm{~cm}$. Horizontal and vertical axes are voltage $(\mathrm{mV})$ and time (ns), respectively

\begin{tabular}{llll}
\hline References & Frequency & Pathloss exponent $(n)$ & Tested body part \\
\hline$[25]$ & $2.45 \mathrm{GHz}$ & 2.16 & Arm \\
{$[26]$} & $6-9 \mathrm{GHz}$ & 4.9 & Arm \\
{$[26]$} & $4-5 \mathrm{GHz}$ & 3.2 & Arm \\
{$[27]$} & $2.45 \mathrm{GHz}$ & 3.47 & Not reported \\
{$[28]$} & $2.45 \mathrm{GHz}$ & 3.11 & Arm \\
{$[29]$} & $2.4 \mathrm{GHz}$ & 2.57 & Not reported \\
{$[30]$} & $20-60 \mathrm{MHz}$ & 1.8 & Not reported \\
This work & $0.5-17.5 \mathrm{MHz}$ & 0.75 & Arm \\
\hline
\end{tabular}
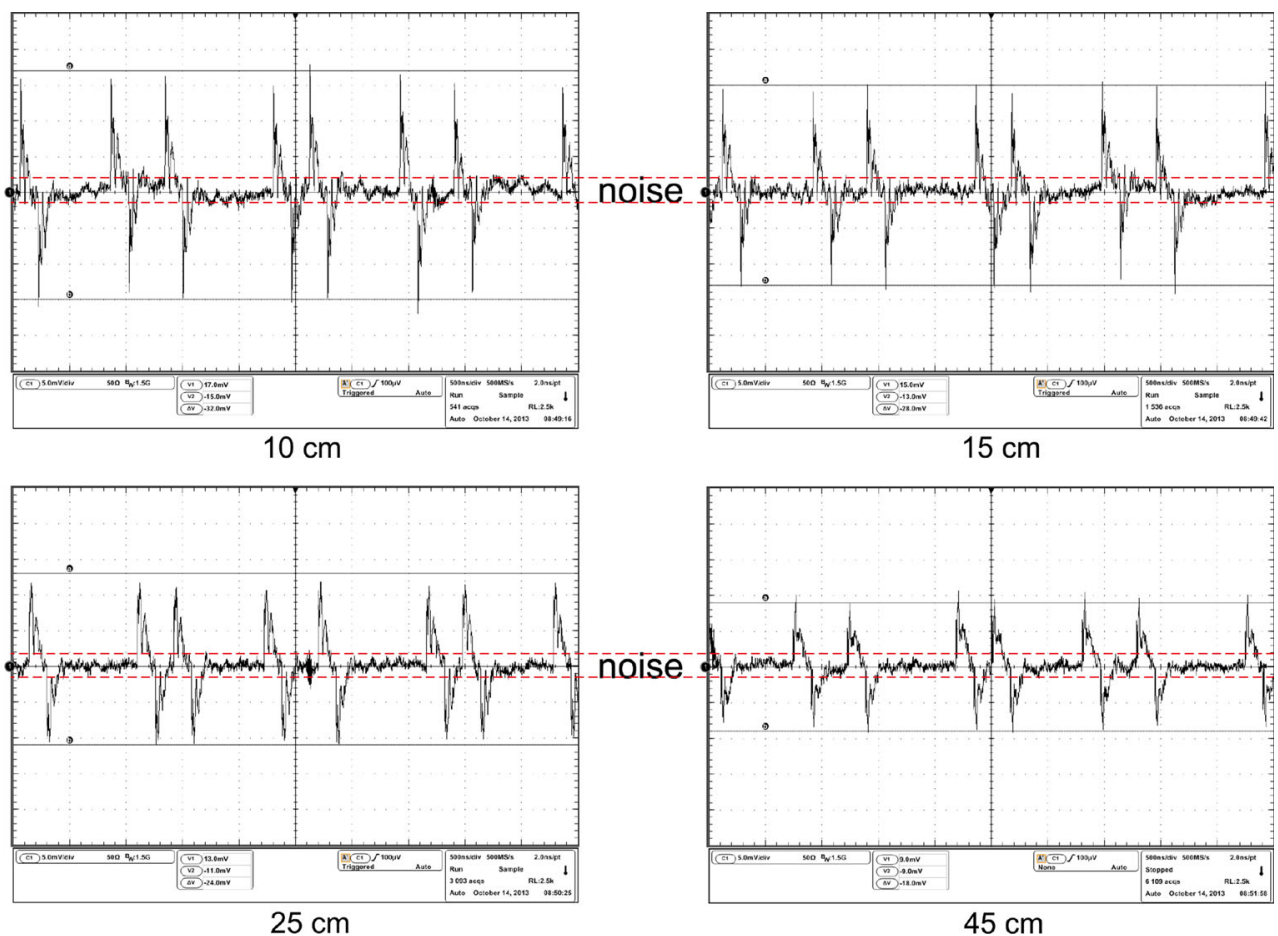

transmission schemes. All curves decrease almost linearly between 5 and $50 \mathrm{~cm}$. The SNR values are about $17.5 \mathrm{~dB}$ after $5 \mathrm{~cm}$ of signal propagation for subject 1 using the 4and 8-PPM schemes. For a communication distance of $50 \mathrm{~cm}$, the SNR values are 13 and $11 \mathrm{~dB}$ for the 4- and 8-PPM schemes, respectively. For both subjects, the 4-PPM scheme always exhibit higher SNR than that of the 8-PPM scheme because of the higher average transmission frequency of the former (see Fig. 4c). For both 4- and 8-PPM schemes, subject 1 has higher SNR than that of subject 2 because the arm of the former had lower impedance due to differences in the ratio of muscle. Additionally, the differences in subject body composition led to different channel paths. This explains the different SNR values between the subjects. In the ranges $5-10 \mathrm{~cm}$ and $30-35 \mathrm{~cm}$, the decreasing rates are different because of the effect of the joint segment. The nearest parts to the wrist $(5-10 \mathrm{~cm})$ and elbow $(30-35 \mathrm{~cm})$ have different body channel characteristics. In addition, the arm is not entirely homogeneous from end to end. These results coincide with previous observations on signal attenuation trends [33].

According to Shikada et al. [13], IR-type IBC transceivers have about $35-\mathrm{dB}$ path loss between 5 and $50 \mathrm{~cm}$ through body tissue. There is around $15 \mathrm{~dB}$ attenuation from 5 to $50 \mathrm{~cm}$ of the human body channel calculated based on the receiver input power of the IBC system with an all-digital-circuit wideband signaling scheme presented by Seong-Jun et al. [34]. In this work, SNR calculation $(5-50 \mathrm{~cm})$ was used to clarify the signal attenuation using the PPM scheme. Due to the variation of channel length from 5 to $50 \mathrm{~cm}$ in subject 1, the SNR varies up to $6.5 \mathrm{~dB}$ for both kinds of PPM. This value was $8.5 \mathrm{~dB}$ for subject 2 (see Fig. 10a). 
Fig. 8 Amplitude of IBC received signal and noise using a 4-PPM scheme and b 8-PPM scheme between 5 and $50 \mathrm{~cm}$ at human arm channel for subject 1
Fig. 9 Analytical model of human body communication channel for various on-body channel lengths

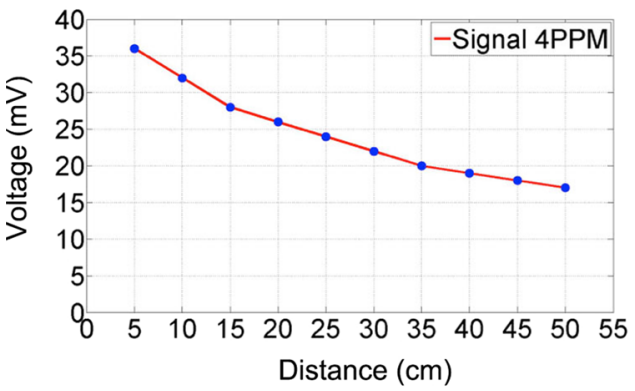

(a)

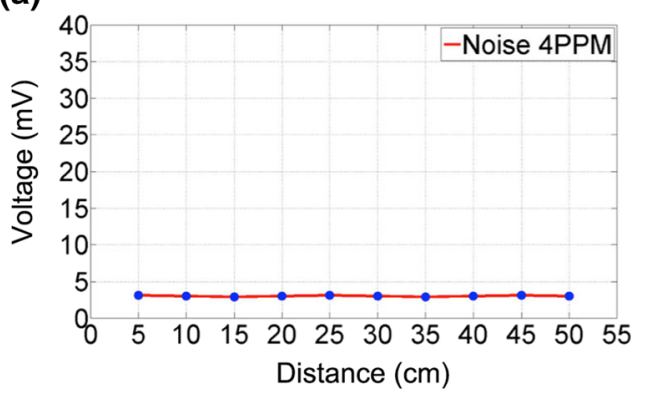

(b)
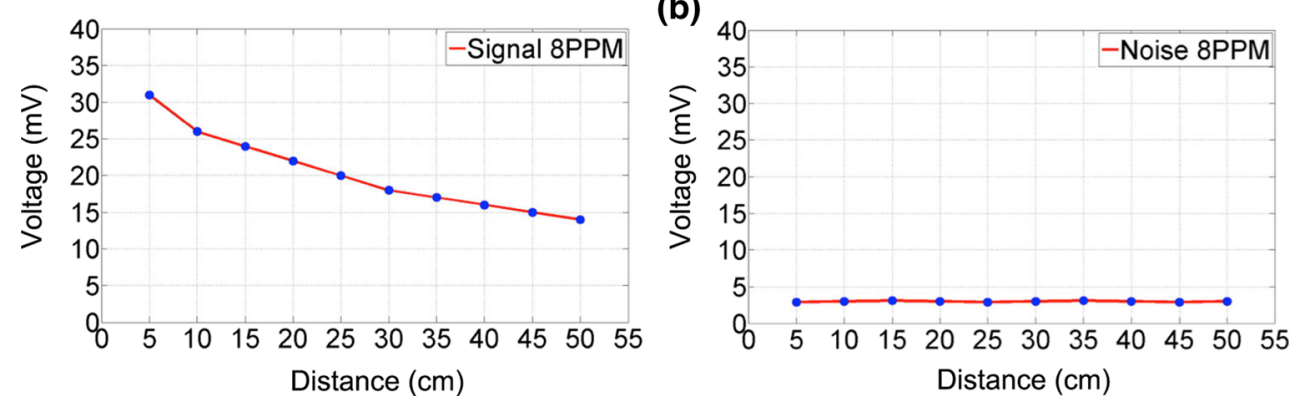
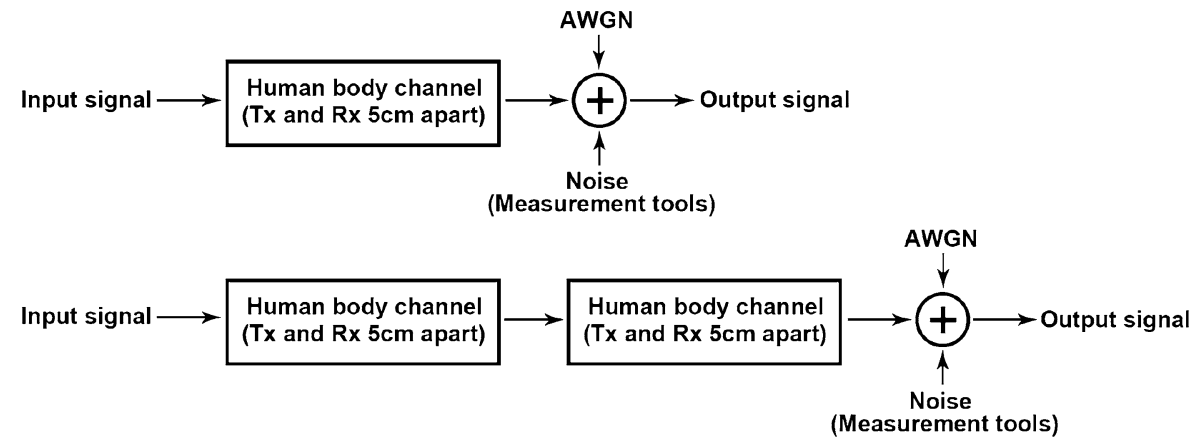

(Measurement tools)

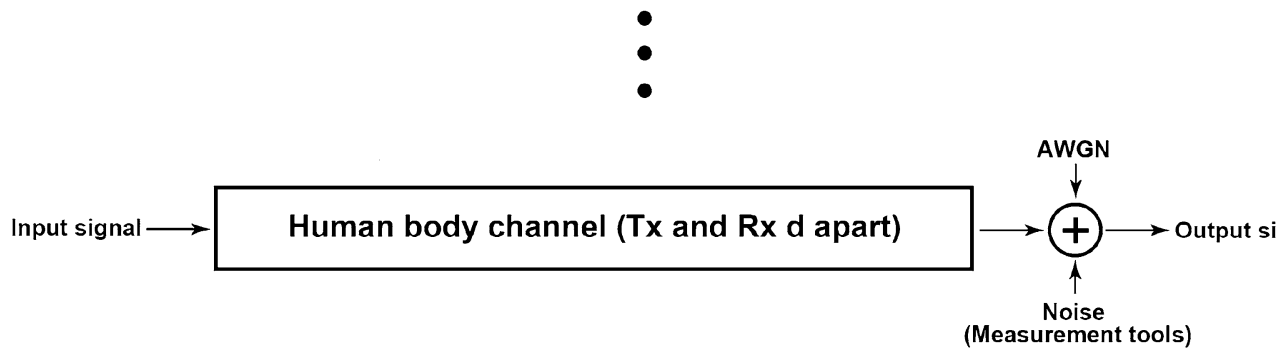

BER is the probability that an error may occur in a bit in the pulse train, where a ' 1 ' bit turns into a ' 0 ' bit or vice versa. Time slot coding is achieved by converting each word of $\mathrm{N}$ bits into one of $\mathrm{L}=2^{\mathrm{N}}$ slices for transmission. Since noise is AWGN, the probability of error can be expressed in terms of the error function. The theoretical BER for an L-PPM scheme is given by [35, 36]:

$B E R_{P P M}=\frac{1}{2} \operatorname{erfc}\left(\frac{1}{2 \sqrt{2}} \sqrt{S N R \frac{L}{2} \log _{2} L}\right)$

where erfc is the complementary error function, expressed as: $\operatorname{erfc}(\mathrm{x})=\left(\frac{2}{\sqrt{\pi}}\right) \int_{x}^{\infty} \exp \left(-t^{2}\right) d t$

Figure 10b shows BER performance versus IBC channel length for the 4- and 8-PPM schemes obtained from a theoretical simulation using Matlab software. Each curve is based on 10 different receiver positions on the body (see Fig. 3). Equation (4) was used to calculate the theoretical result of BER by linking the SNR to the corresponding distance via the measured path loss during signal transmission through the body. The plot shows how the BER 


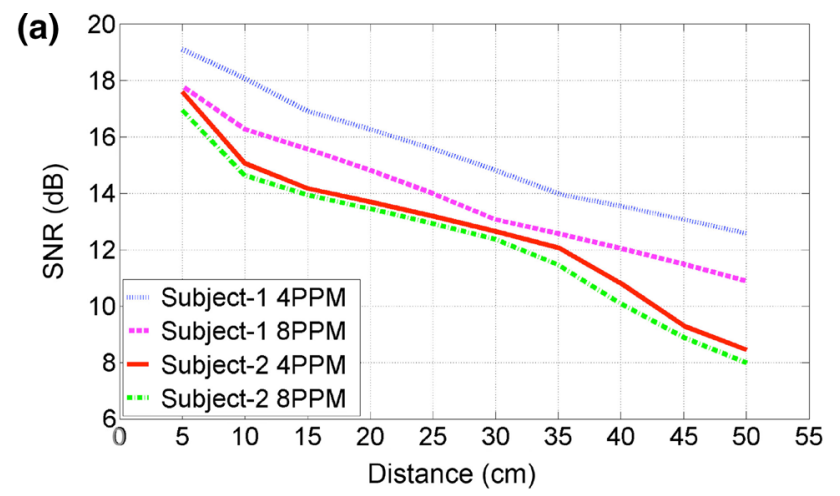

(b)

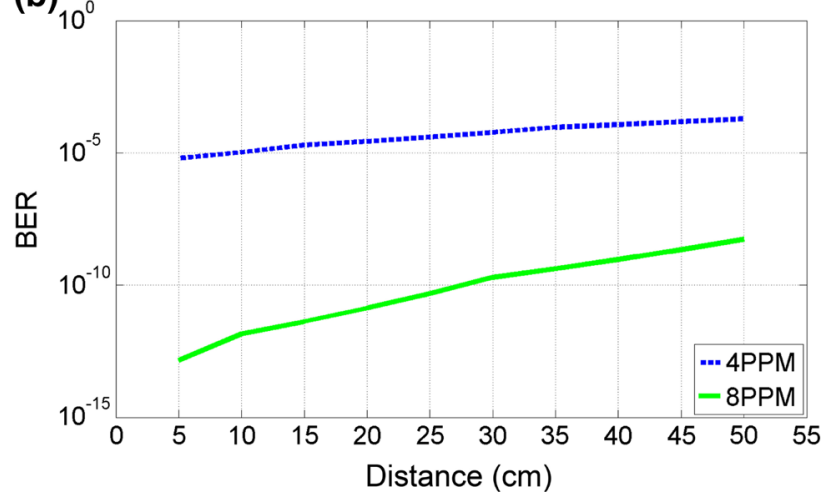

Fig. 10 a SNR versus channel length or distance $(5-50 \mathrm{~cm})$ using 4and 8-PPM IBC system for subjects 1 and 2. b Simulation result of BER versus distance for $4-$ and 8-PPM schemes

degrades as a function of distance. For a fixed distance, the BER performance for the 4-PPM scheme is better than that for the 8-PPM scheme. Furthermore, as the distance increase, the BER performance gets worse due to the corresponding increase in path loss in biological tissues. A similar analysis was conducted by Timmermann et al. [9], who evaluated the indoor RF system performance of an IR transmission system in terms of BER versus channel distance. The BER result of their RF system is similar to that of the 4-PPM scheme in our study. The 8 PPM or higher L-PPM has the potential to achieve better performance comparing to the current RF short-range communication system. The 4- and 8-PPM schemes had BER values of less than 0.001 , which is an acceptable value in digital communication [33].

\section{Conclusion}

This paper presented an IR-IBC system based on PPM schemes for signal transmission along the human arm. The PPM scheme (4- and 8-PPM) is a novel modulation method for IBC system design. We examined the path loss during communication through fixed channel lengths with galvanic coupling IBC. The channel noise, SNR, and BER of the IBC transmitter implemented on an FPGA platform were measured. The results indicate that the SNR and BER degrade as a function of communication channel length. The path loss characteristics were formulated and the relationship between the value of the body path loss exponent and the operation frequency was considered. The 8-PPM scheme is a better option for IBC, as it balances BER and SNR. The proposed PPM IBC system can enhance the performance for sensor networks in biomedical applications.

\section{References}

1. Niemela, V., Hamalainen, M., \& Iinatti, J. (2013). On IEEE 802.15.6 UWB symbol length for energy detector receivers' performance with OOK and PPM. In Proceedings of the 7th international symposium on medical information and communication technology (ISMICT), 2013, pp. 33-37.

2. Kim, J., Song, I., \& Choi, E. J. S. (2012). A dynamic duty cycle MAC algorithm for wireless body area networks. International Journal of Bio-Science and Bio-Technology, 4, 83-92.

3. Smith, D., Dino, M., Tharaka, L., \& Leif, H. (2013). Propagation models for body area networks: a survey and new outlook. IEEE Antennas and Propagation Magazine, 55, 97-117.

4. IEEE Standard for Local and Metropolitan Area Networks-Part 15.6: Wireless Body Area Networks, IEEE Std. 802.15.6TM2012, Feb. 2012.

5. Movassaghi, S., Mehran, A., Justin, L., Smith, D., \& Jamalipour, A. (2014). Wireless body area networks: A survey. IEEE Communications Surveys \& Tutorials, 16(3), 1-29.

6. Seokseong, J., Yu, C., Suh, Y., Moon, J., \& Lee, S. (2011). Analysis of body communication parameters using Software Radio platform. In Proceedings-IEEE military communications conference (MILCOM), 2011, pp. 628-633.

7. Zimmerman, T. G. (1995). Personal area networks (PAN): nearfield intra-body communication. IBM Systems Journal, 35(3\&4), 609-617.

8. Bae, J., \& Yoo, H. J. (2015). The effects of electrode configuration on body channel communication based on analysis of vertical and horizontal electric dipoles. IEEE Transactions on Microwave Theory and Techniques, 63(4), 1409-1420.

9. Timmermann, J., Pancera, E., Adamiuk, G., Wiesbeck, W., \& Zwick, T. (2008). Estimated performance of UWB impulse radio transmission including dirty RF effects. In Proceedings of the IEEE conference ultra-wideband (ICUWB), 2008, pp. 205-208.

10. Anzai, D., Katsu, K., Chavez-Santiago, R., Wang, Q., Plettemeier, D., Wang, J., et al. (2013). Experimental evaluation of implant UWB-IR transmission with living animal for body area networks. IEEE Transactions on Microwave Theory and Techniques, 61(1), 183-192.

11. Zhang, L., Liu, X., Huang, J., \& Wang, L. (2010). Baseband system for human body channel communication. In Proceedings of the Biomed. Eng. Inform. (BMEI), 2010, pp. 778-781.

12. Tengfei, L., Zedong, N., Wenchen, W., Feng, G., \& Lei, W. (2011). A human body communication transceiver based on onoff keying modulation. In Proc. Int. Symp. Bioelectron. Bioinform. (ISBB), 2011, pp. 61-64.

13. Shikada, K., \& Jianqing, W. (2012). Development of human body communication transceiver based on impulse radio scheme. In Proc. 2nd IEEE CPMT Symp., 2012, Japan, pp. 1-4. 
14. Seyedi, M. H., \& Lai, D. T. H. (2014). Effect of limb joints and limb movement on intrabody communications for body area network applications. Journal of Medical and Biological Engineering (JMBE), 34(3), 276-283.

15. Seyedi, M. H., Kibret, B., Lai, D. T. H., \& Faulkner, M. (2013). A survey on intrabody communications for body area network applications. IEEE Transactions on Biomedical Engineering, 60, 2067-2077.

16. Hämäläinen, M. (2006). Singleband UWB systems: Analysis and measurements of coexistence with selected existing radio systems. Acta Universitatis Ouluensis, Series C, Technica 240, University of Oulu, 2006.

17. Fu, X., Chen, G., Tang, T., Zhao, Y., Wang, P., \& Zhang, Y. (2010). Research and simulation of PPM modulation and demodulation system on spatial wireless optical communication. In Proc. Symp. Photon. Optoelectron. (SOPO), 2010, pp. 1-5.

18. Seyedi, M. H., Cai, Z., Lai, D. T. H., \& Rivet, F. (2014). An energy-efficient pulse position modulation transmitter for galvanic intrabody communications. In International conference on wireless mobile communication and healthcare, Greece, 3-5 Nov. 2014, pp. 192-195.

19. International Commission on Non-Ionizing Radiation Protection. (1998). Guidelines for limiting exposure to time-varying electric, magnetic, and electromagnetic fields (up to $300 \mathrm{GHz}$ ). Health Physics, 74(4), 494-522.

20. Swaminathan, M., Cabrera, F., Pujol, J., Muncuk, U., Schirner, G., \& Chowdhury, K. R. (2015). Multi-path model and sensitivity analysis for galvanic coupled intra-body communication through layered tissue. IEEE Transactions on Biomedical Circuits and Systems, 1 .

21. Takumi, K., Shimatani, Y., \& Masaki, K. (2012). Application of near-field intra-body communication and spread spectrum technique to vital-sign monitor. In Proceedings of the 32th annual international conference IEEE EMBC, 2012, pp. 4517-4520.

22. Friis Transmission Equation. [Online]. Available: http://en.wiki pedia.org/wiki/Friis_transmission_equation

23. Pham, T. V., Kim, Y. T., Thi, M. T., \& Tuy, H. A. (2010). An empirical channel model for Human Body Communication. Advanced Technologies for Communications (ATC), 352(355), 20-22.

24. Ai, Y., Tao, W., \& Choy, C. (2012). A simulation-oriented channel modeling methodology for human on-body channel communication. In Proceedings of the emerging application of information technology (EAIT), 2012, pp. 73-76.

25. Rahim, H. A., Malek, F., Ahmad, A. I., \& Malek, M. F. Path loss characterization of body-to-body radio propagation channel on the angular variations. Session 1PK: 399.
26. Wang, H. (2010). Wireless body area networks path loss characterization analysis. In Proc. Int. Conf. Comput. Engin. Technol. (ICCET), pp. V2-163.

27. Kikuzuki, T., Andrenko, A. S., Hossain, M. G., Ida, I., Kasai, K., \& Ohashi, Y. (2011). A simple path loss model for body area network in the bed side monitoring applications. In Proceedings of the Asia-Pacific microwave conference (APMC), 2011, pp. 765-768.

28. Reusens, E., Wout, J., Benoît, L., Bart, B., Günter, V., Emmeric, T., et al. (2009). Characterization of on-body communication channel and energy efficient topology design for wireless body area networks. IEEE Transactions on Information Technology in Biomedicine, 13(6), 933-945.

29. Guraliuc, A., Serra, A., Nepa, P., Manara, G., Potorti, F., \& Barsocchi, P. (2009). Body posture/activity detection: Path loss characterization for $2.4 \mathrm{GHz}$ on-body wireless sensors. In Proc. Inter. Symp. Antenna. Propag. Society, 2009, pp. 1-4.

30. Shi, J., \& Wang, J. (2010). Dual-mode impulse radio ultrawideband transmission for body area networks. IET Microwaves, Antennas \& Propagation, 5(10), 1250-1255.

31. Kobayashi, T., Sugimoto, C., \& Kohno, R. (2014). Performance evaluation of the spread spectrum human body communication devices. In Proceedings of the medical information and communication technology (ISMICT), 2014, pp. 2-4.

32. Wegmueller, M. S. (2007). Intra-body communication for biomedical sensor networks. Ph.D. dissertation, ETH Zurich, Zurich, Switzerland.

33. Seyedi, M. H., Lai, D. T. H., \& Faulkner, M. (2012). Limb joint effects on signal transmission in capacitive coupled intra-body communication systems. In Proceedings of the 34th annual international conference of the IEEE engineering in medicine and biology society, San Diego, CA, Aug. 2012, pp. 6699-6702.

34. Seong-Jun, S., Namjun, C., Sunyoung, K., \& Hoi-Jun, Y. (2007). Energy-efficient human body communication receiver chipset using wideband signaling scheme. In Proceedings of the 29th annual international conference of the IEEE engineering in medicine and biology society, 2007, pp. 2292-2295.

35. Elganimi, T. Y. (2013). Performance comparison between OOK, PPM and PAM modulation schemes for free space optical (FSO) communication systems: Analytical study. International Journal of Computer Applications (0975-8887), 79(11), 22-27.

36. Manea, V., Dragomir, R., \& Puscici, S. (2011). OOK and PPM modulations effects on bit error rate in terrestrial laser transmissions. TELECOMUNICAŢII, Anul LIV, nr. 2/2011, pp. 55-61. 